\title{
Comparison of engine/inlet distortion measurements with MEMS and ESP pressure sensors
}

\author{
AIAA-2004-2398 \\ Hector L. Soto* \\ NASA Langley Research Center \\ Hampton, Virginia \\ Corey D. Hernandez ${ }^{\dagger}$ \\ Swales Aerospace, Inc. \\ Hampton, Virginia
}

\begin{abstract}
A study of active-flow control in a small-scale boundary layer ingestion inlet was conducted at the NASA Langley Basic Aerodynamic Research Tunnel (BART). Forty MEMS pressure sensors, in a rake style configuration, were used to examine both the mean (DC) and high frequency (AC) components of the total pressure across the inlet/engine interface plane. The mean component was acquired and used to calculate pressure distortion. The AC component was acquired separately, at a high sampling rate, and is used to study the unsteady effects of the active-flow control. An identical total pressure rake, utilizing an Electronically Scanned Pressure (ESP) system, was also used to calculate distortion; a comparison of the results obtained using the two rakes is presented.
\end{abstract}

\section{Introduction}

The efficiency of an aircraft's engine is greatly impacted by its placement on the surface of the vehicle. In NASA's design of the Blended Wing Body (BWB), the optimal location for engines and inlets is near the upper surface on the aft section of the vehicle. Unfortunately, incorporation of the inlet on the surface of the vehicle increases the technical risk of the configuration ${ }^{1}$. The NASA Ultra Efficient Engine Technology (UEET) program chose to pursue the use of boundary layer ingestion (BLI) inlets with an active flow control system for the BWB design to reduce this risk. Different inlets have been studied, from semi-elliptical geometries ingesting boundary layers with a height on the order of $30 \%$ of the inlet diameter to rectangular inlets ingesting $150 \%$ of the airfoil's boundary layer height. It has been shown that the use of these inlet designs may potentially increase the aircraft's cruising distance by $10 \%$, if one was able to control the adverse effects of this design in terms of distortion and pressure recovery ${ }^{2}$. The inlet presented in this study incorporates a cylindrical s-inlet with $30 \%$ BLI.

The UEET program has been studying different methods to reduce the distortion caused by the aforementioned BLI inlet. In this test, an active flow control system utilizing high-pressure air was positioned upstream of the engine inlet to control pressure distortion. This method was shown to reduce the $29 \%$ baseline distortion value to around $7 \%$ as measured using the DC60 calculation method ${ }^{3}$. This paper presents the results of using MEMS pressure sensors to measure the total pressure used to calculate distortion, and how these results compare to the wind tunnel standard ESP systems.

Additionally, the MEMS sensors are used to measure the high frequency response of the flow when the active flow control system was in operation, with the main objective being to observe the effects of the jets or the active flow control system at the engine/inlet interface.

\footnotetext{
* Research Engineer, Advanced Model and Sensor Systems Branch

${ }^{\dagger}$ Research Engineer, Advanced Sensing and Optical Measurements Branch
} 


\section{Measurement setup}

The MEMS sensors utilized in the test are commercial off the shelf (COTS) products utilizing an active four-arm strain gage Wheatstone bridge configuration. The sensors resonance frequencies are on the order of $70 \mathrm{kHz}$ and calibrations show that their frequency responses were flat from $50 \mathrm{~Hz}$ to $14 \mathrm{kHz}$. Additionally, the sensors are temperature compensated and feature a pressure range of $+/-2$-psid. Other sensor characteristics include a sensitivity of $150 \mathrm{mV} / \mathrm{psi}$ (with a $10 \mathrm{~V}$ excitation), and a tip diameter of 0.092 inches. Each differential pressure sensor is subsequently tip mounted in 1/8-inch diameter steel tubing. The forty sensors are then mounted in a rake configuration (Fig 1), following the aerospace recommended practice, ARP 1420.

A signal conditioner provided a pre-filter gain of 64, and the signal was low-pass filtered with a 4pole Time Delay (TD) filter with a cutoff frequency of $5 \mathrm{kHz}$. A post filter gain was set to 2.34 , for a total gain of 150 . All the reference ports are interfaced to a manifold and referenced to atmosphere.

Two data acquisition systems were used to acquire the DC and AC components separately but simultaneous of the pressure signal. This method is chosen to maximize the gain and so as not to saturate the data acquisition system. Additionally, this allowed the users to maximize the 16 bits of resolution over the full scale of the signal. Sampling rates used for acquisition were $200 \mathrm{~Hz}$ for the DC component and $12.8 \mathrm{kHz}$ for the AC component. For each condition in the test matrix eight seconds of data are acquired yielding 1600 and 102,400 samples for the DC and $\mathrm{AC}$ data respectively.

\section{Calibration}

Pre-test, in situ and post-test calibrations were conducted and the data was compared. The calibration was conducted using a $+/-2$ psi pressure controller with $0.003 \%$ FS precision and $0.01 \%$ FS accuracy. All sensors were first zero balanced using the signal conditioner features prior to any calibration. During the test, a calibration was performed prior to acquiring any data and following any changes in the test configuration. Additionally, a dynamic calibration was conducted prior to the test on each individual sensor using sound pressure levels.

\section{Experimental setup}

The test was conducted at the NASA Langley Research Center in the Basic Aerodynamic Research Tunnel (BART) on a $6.129 \%$ BLI inlet model. The maximum achievable flow velocity in the test section is approximately Mach 0.17. The inlet to the s-duct begins 50 inches downstream of the start of the test section. A figure of the entire setup is shown in figure 2. This placement allows the tunnel's natural boundary layer to be nominally $30 \%$ of the inlet's diameter when it reaches the entrance to the inlet. The boundary layer height for this configuration was verified prior to the test using Stereo Digital Particle Image Velocimetry (SDPIV). For further details on the construction of the inlet, development and use of the active flow control system, and the SDPIV work the reader is referred to reference 2 .

\section{Test Conditions}

For this test all runs were conducted at a tunnel Mach number of 0.15 . The test matrix consisted of setting the sinlet mass flow to 1800 standard cubic feet per minute (SCFM) and varying the mass flow through the actuators between zero and the maximum achievable mass flow. Total pressure data was taken from the MEMS rake at all of these conditions. The test matrix values were determined earlier in the wind tunnel test by evaluating the results of the ESP rake as outlined in reference 2 . 


\section{Discussion of Results}

Data was obtained for the MEMS and ESP total pressure rakes separately. The tunnel and other conditions for which data was collected for both rakes were matched as closely as possible. Repeating exact tunnel conditions is very difficult, however, in order to make a better comparison of the two rakes, pressure coefficients (Cp) are calculated and then compared, as shown in Figure 3.

$$
C p=\frac{(P t-P s)}{q}
$$

Where Pt is the measured total pressure from the MEMS rake, Ps is the tunnel static pressure and $\mathrm{q}$ is the tunnel dynamic pressure.

As seen in the plot, the data tracks very nicely for most sensors. Some of the minor discrepancies for three of the sensors are explained via a minor sensor misalignment with respect to the flow. Following the test it was discovered that three sensor housings (one sensor on the MEMS rake (sensor \#40) and two sensors on the ESP rake (sensors \#38 and \#39)) became slightly bent during installation. Since the DC45 distortion calculation is based on the average reading of the 40 sensors, this difference in measured pressure values only minimally affected the calculated distortion value ${ }^{3}$. This artifact is seen in Figure 4a. Figure 4b shows a comparison of the MEMS and ESP DC60 and DC45 for varying actuators mass flow. The DC60 for this comparison was around $2 \%$ higher than the calculated distortion, but for the DC45 it was within 0.5\% except for high actuators mass flow conditions. This pattern was observed on all the conditions compared. A comparison of the Cps for every clock position was analyzed and the results are shown in figure 3. As you can observe the Cps for the zero degree and thirty degree positions are in agreement. In the case of the fifteen degree position and actuators at a high SCFM the Cps don't agree. The reason for this disagreement is not well understood and further research is needed.

Analysis of the sensors AC signal, as seen in figure 5, shows that the peak to peak voltage generated by the active flow control, with tunnel and blower off, is more than one order of magnitude less than when the tunnel and the blower were being operated at maximum tested condition. All other sensors showed similar results.

\section{Conclusion}

MEMS dynamic pressure sensors may be used as a substitute to the ESP measurement system. The sensors showed that they could provide time-dependent data and total pressure simultaneously. They also showed that they could make these measurements without increasing inlet blockage over the ESP system.

The advantage of the MEMS sensor over the ESP are mentioned above, but the disadvantage of this system is cost. In this case the cost of the sensors, signal conditioner, and data acquisition was much higher than an ESP system.

The MEMS sensor data closely matched the ESP sensor data for the conditions tested. Any extrapolation to different test conditions or system setup should be done with caution. To support this research, the researchers would like to conduct more studies to determine the effects of flow angularity and higher flow rates on these types of sensors.

\section{References}

1. Liebeck, R. H.,"Design of the Blended-Wing-Body Subsonic Transport," AIAA 2002-002.

2. Susan Althoff Gorton, Lewis R. Owens, Luther N. Jenkins, "Active Flow Control on a Boundary-LayerIngesting Inlet", AIAA 2004 42nd Aerospace Sciences Conference, accepted for publication, 2004

3. Seddon, J., and Goldsmith, E. L., Intake Aerodynamics, Second Edition, AIAA, Reston, Virginia, 1999, p. 169, 278-280 
Figures
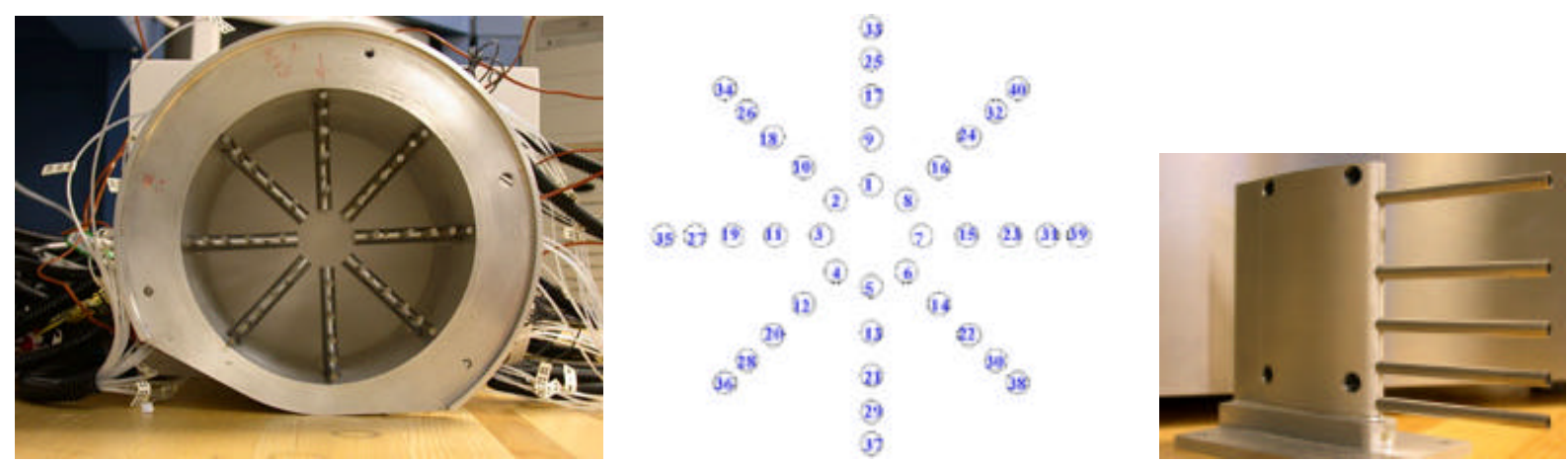

Figure 1: Rake and model at engine interface plane, and corresponding sensor numbers

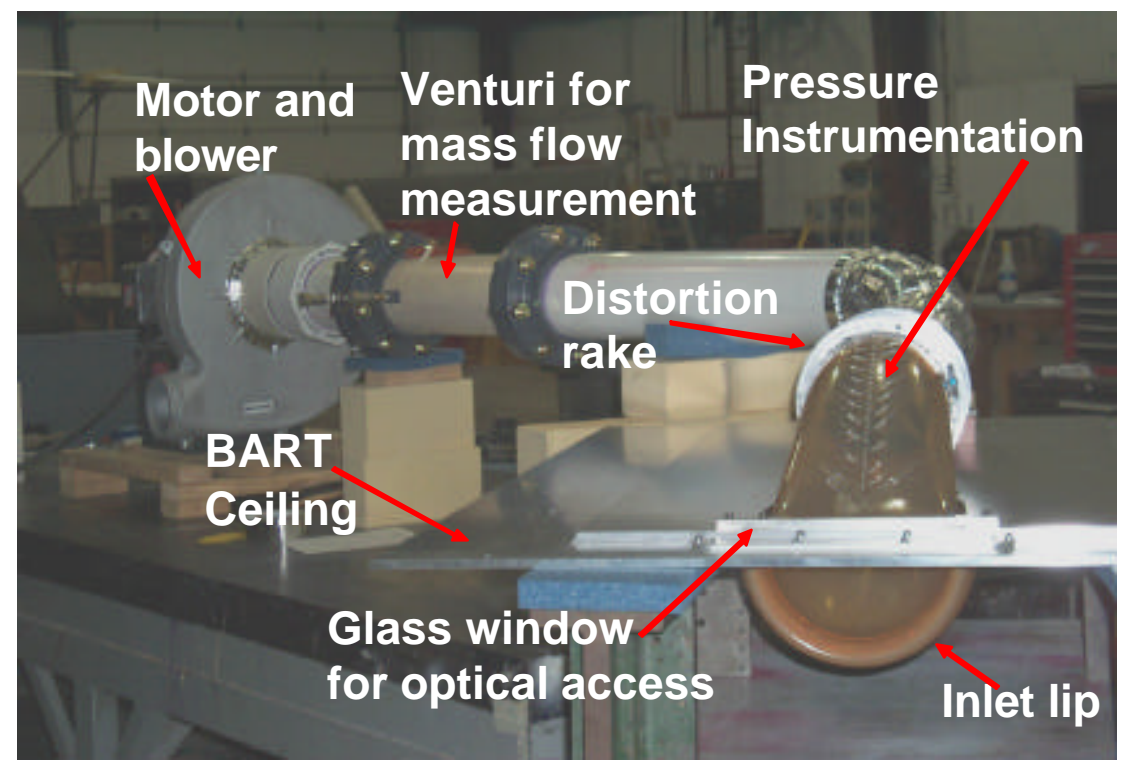

Figure 2: Complete model used at the BART 

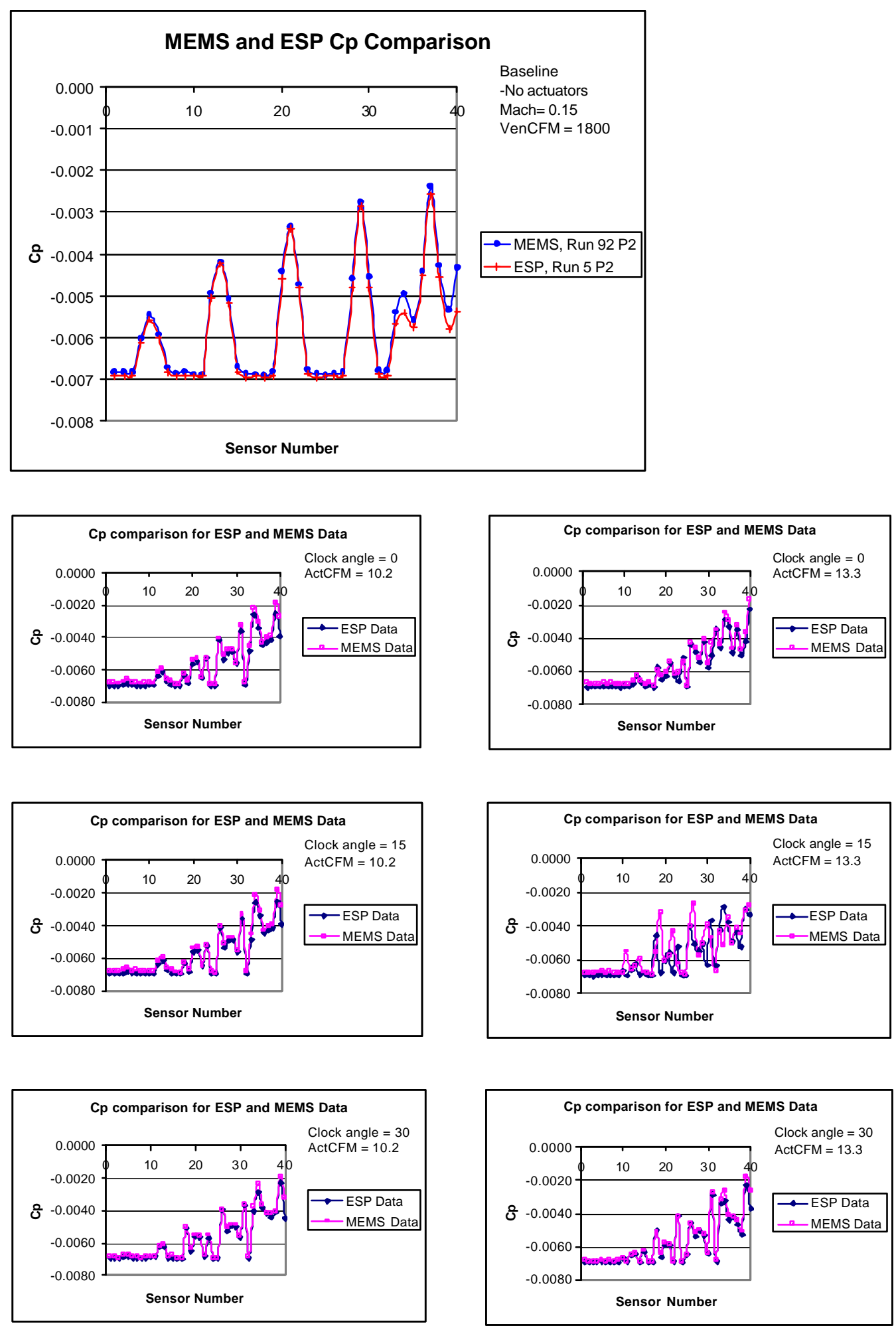

Figure 3: Comparison of $\mathrm{Cp}$ values calculated using the MEMS and ESP total pressure rake data. 


\section{Pressure data for MEMS and ESP sensor rake comparisons}
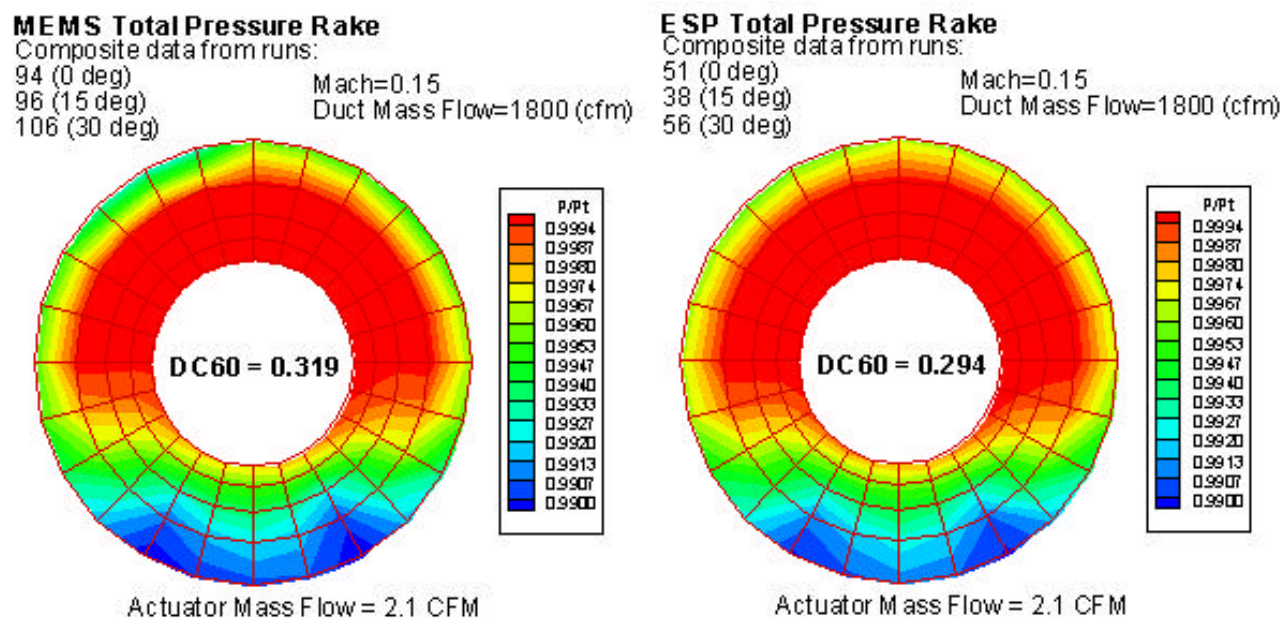

Actuator M ass Flow $=2.1 \mathrm{CFM}$
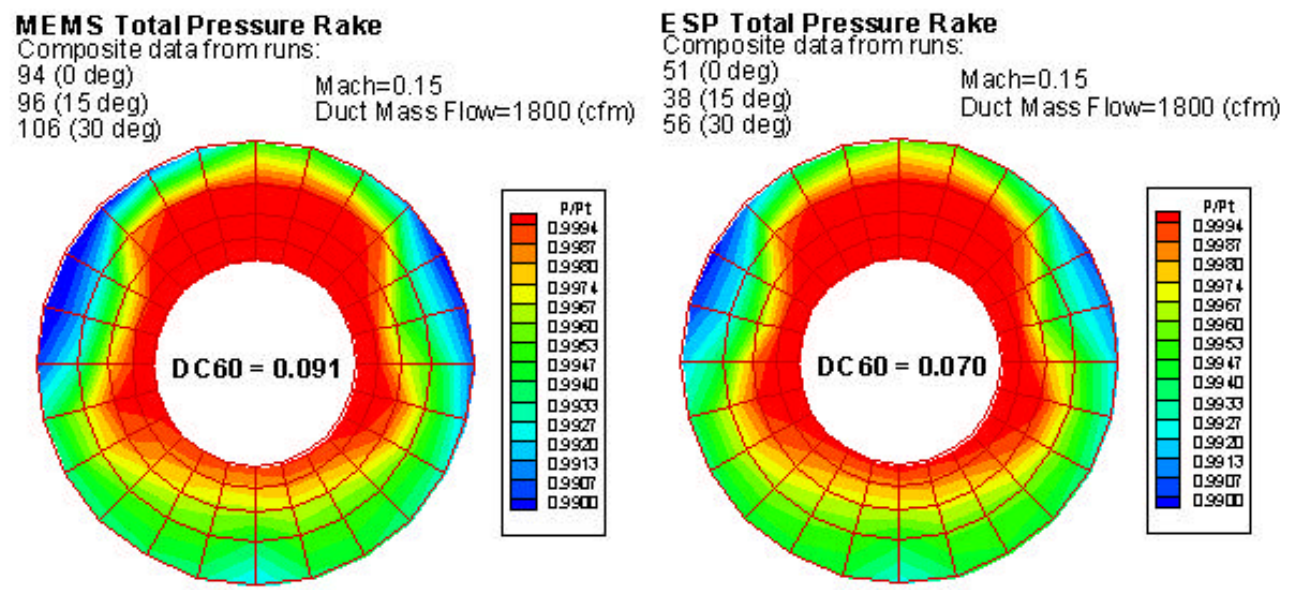

Actuator Mass F low $=10.2 \mathrm{CF} \mathrm{M}$

Actuator M ass Flow $=10.2 \mathrm{CFM}$

Figure 4a: Comparison of pressure ratios and distortion values calculated using the MEMS and ESP total pressure rake data. 

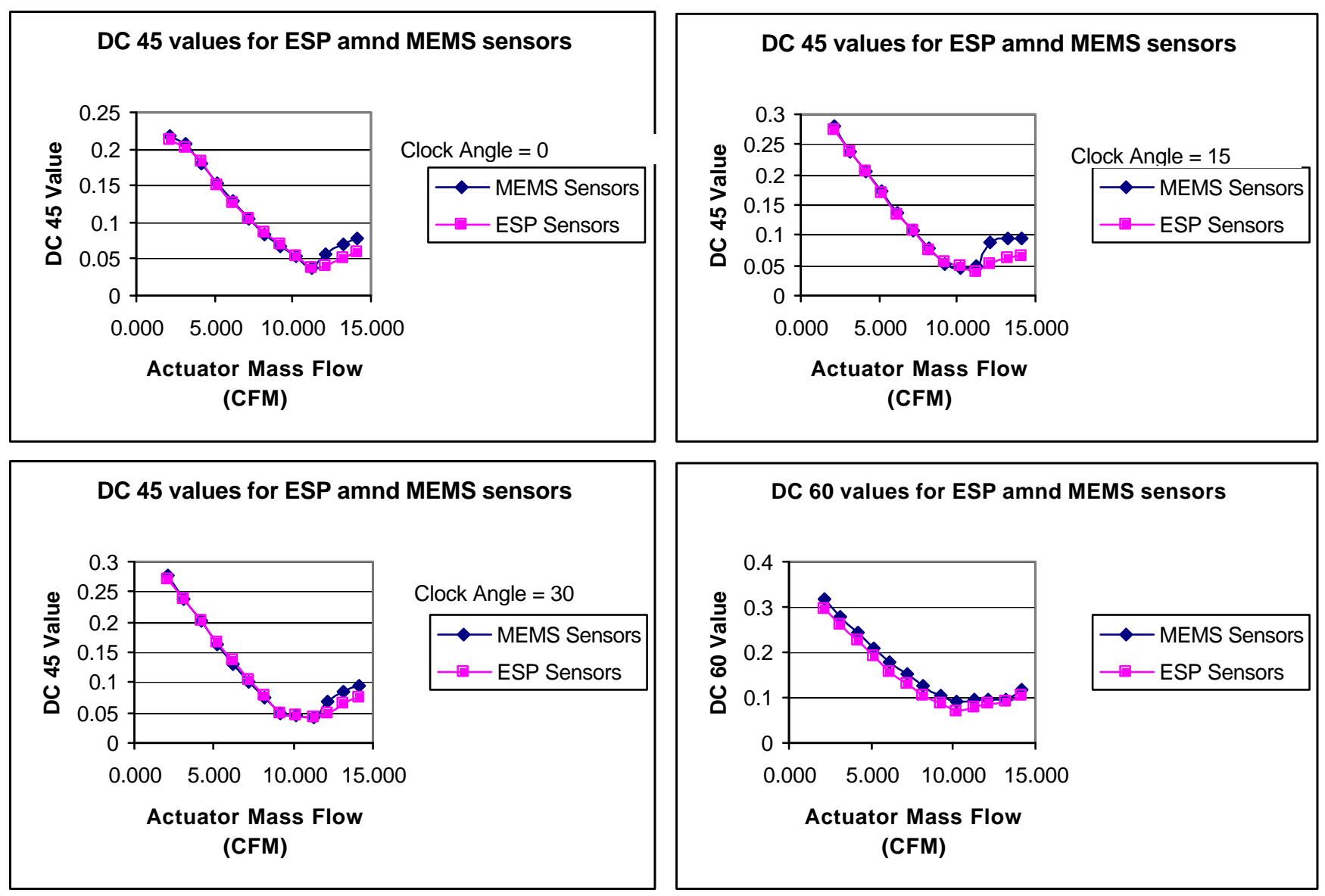

Figure 4b: Comparison of distortion values calculated using the MEMS and ESP total pressure rake data.

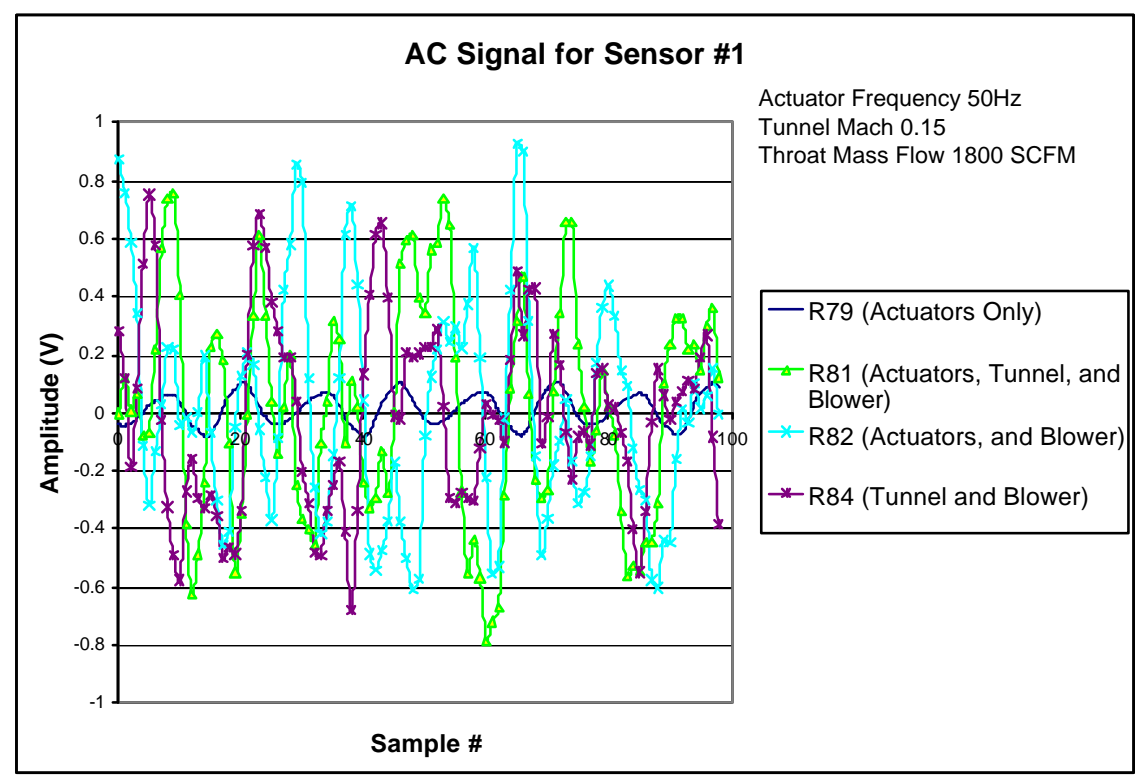

Figure 5: Typical AC signal for the MEMS pressure sensors. 